\title{
La experimentación de sí mismo y los juegos de verdad. Una aproximación al Movimiento Quantified Self.
}

\section{Self-experimentação e jogos de verdade. Uma abordagem ao Movimento do Quantified Self.}

\section{Self experimentation and the games of truth. An approach to the Quantified Self Movement.}

Diego González García; Marco Maureira Velasquez; Francisco Tirado Serrano

Universidad de la República, Uruguay; Universidad de Barcelona, España; Universidad Autónoma de Barcelona, España

\section{RESUMEN:}

El presente artículo presenta una revisión de las principales líneas de reflexión e investigación sobre el movimiento Quantified Self. Dentro de este amplio abaníco de propuestas, nos centraremos en lo que denominaremos perspectivas críticas, las cuales serán agrupadas en tres grandes movimientos: a) gubernamentalidad y biopolítica; b) estudios postfenomenológicos; c) nuevos materialismos. Argumentamos que cada uno de estos enfoques enfatiza (de forma prioritaria, no exclusiva) el ejercicio de un determinado juego de verdad: la "verdad disciplinar" de la gubernamentalidad, la "verdad de sí" de la post-fenomenología y la "verdad técnica" de los nuevos materialismo. Sin embargo, la experimentación de si mismo que despliega el movimiento $Q S$ implica una puesta en juego simultánea de estas tres dimensiones, razón por la cual crearemos un dispositivo conceptual que permita dar cuenta de estos juegos de verdad de forma conjunta: la tecnoaleturgia.

Palabras-clave: juegos de verdad; experimentación de sí; quantified self

\section{RESUMO:}

Este artigo apresenta uma revisão das principais linhas de reflexão e investigação sobre o Movimento do Quantified Self. Dentro desta vasta gama de propostas, focaremos em aquilo a que chamaremos perspectivas críticas, que serão agrupadas em três movimentos principais: a) governabilidade e biopolítica; b) estudos pós-fenomenológicos; c) novos materialismos. Argumentamos que cada uma destas abordagens enfatiza (como prioridade, não exclusivamente) o exercício de um certo jogo de verdade: a "verdade disciplinar" da governabilidade, a "verdade do self" da pós-fenomenologia e a "verdade técnica" dos novos materialismos. Contudo, a experimentação do self que o movimento $Q S$ implanta implica um jogo simultâneo destas três dimensões, razão pela qual criaremos um dispositivo conceptual que nos permite dar conta destes jogos de verdade em conjunto: a tecnoaleturgía.

Palavras-chave: jogos de verdade; experimentação do self; quantified self 


\begin{abstract}
:
This article presents a review of the main lines of reflection and research on the Quantified Self movement. Within this wide range of proposals, we will focus on what we will call critical perspectives, which will be grouped into three main movements: a) governmentality and biopolitics; b) post-phenomenological studies; c) new materialisms. We argue that each of these approaches emphasises (as a priority, not exclusively) the exercise of a particular set of truth: the "disciplinary truth" of governmentality, the "truth of the self" of postphenomenology and the "technical truth" of the new materialisms. However, the experimentation of the self that the QS movement deploys implies a simultaneous play of these three dimensions, which is why we will create a conceptual device that allows us to account for these truth games together: technoallurgy.
\end{abstract}

Key-words: truth games; experimentation of the self; quantified self

DOI:10.12957/mnemosine.2021.62169

\title{
Introducción
}

Somos experimentos jtengamos el valor de serlo!

Nietzsche, Aurora

El movimiento Quantified Self $(Q S)$ es una agrupación internacional integrada por usuarios y fabricantes de herramientas, dedicados a la experimentación y al monitoreo del yo a través de la producción y análisis de datos digitales. Su slogan/objetivo es enfático y directo: Self Knowlege Throught the Numbers. En este sentido, el movimiento QS ha suscitado un enorme interés por parte de las ciencias sociales. Entre la gran diversidad de propuestas, podemos encontrar tres principales enfoques que ofrecen una mirada crítica respecto al desarrollo de este movimiento. En primer lugar, encontramos una serie de trabajos sustentados en las ideas foucoultianas de gubernamentalidad y biopolítica (AJANA, 2017; BEER, 2014; DE SOUZA, 2013; GORDO LÓPEZ, RIVERA; LÓPEZ LOSADA, 2013; LUPTON, 2017; NAFUS; SHERMAN, 2014; SANDERS, 2017). El principal interés de estos trabajos radica en problematizar las consecuencias e implicaciones que las tecnologias de monitorización y producción de datos digitales tienen en nuestras sociedades actuales.

En segundo lugar, podemos destacar la existenca de un campo de investigación que centra su atención en las relaciones entre sujeto y tecnología. La consciencia humana, irremisiblemente ensamblada a nuevos artefectos tecnológicos, ve radicalmente ampliada su capacidad de afección y experimentación de nuevos fenómenos. En este sentido, los estudios 
realizados desde la post-fenomenología (KRISTENSEN; PRIGGE, 2018; KRISTENSEN; RUCKENSTEIN, 2018; VAN DEN EEDE, 2015), centran su preocupación en las experiencias de los usuarios que utilizan dispositivos self-tracking, sosteniendo que dichas relaciones intervienen como mediadores en las formas en que los sujetos se perciben a sí mismos y a su entorno, creando un nuevo ensamblaje entre máquina y ser humano.

Por último, dentro de los Estudios Sociales de la Ciencia y la Tecnología (HARAWAY, 1991; LATOUR, 2008; MOL, 2007), podemos encontrar una preocupación creciente por analizar el movimiento $Q S$ desde un enfoque neo-materialista. Deborah Lupton (2016; 2017), por ejemplo, hace especial hincapié en la utilización de diferentes perspectivas sociomateriales (materialismo feminista, vitalista y antropológico-cultural), con el fin de dar cuenta del ensamblaje entre humanos y dispositivos técnicos que generan datos digitales cada vez más significativos en la vida cotidiana de las personas. En este sentido, se enfatiza la importancia que las "material dimensions of human-data assemblages as they are made, grown, enacted, articulated and incorporated" (LUPTON, 2017: 1).

Ahora bien, lo que aquí sostendremos serán dos importantes cuestiones. Primero, que cada uno de estos enfoques teóricos tiende a privilegiar una cierta mirada: social (en el caso de la gubernamentalidad y la bioplítica), individual (en la post-fenomenología), y una visión más centrada en la materialidad técnica (característica de los nuevos materialismos). Esto, sin embargo, no quiere decir que cada uno de estos enfoques teóricos aprehenda su objeto de estudio de un modo excluyente y exclusivo. Antes bien, lo social, lo individual y lo técnico se encuentran siempre presentes en cada una de las propuestas, sólo que variando su protagonismo. En segundo lugar, sostendremos que dichos protagonismos guardan relación con la puesta en marcha de tres juegos de verdad diferenciados: una "verdad disciplinar" (enfocada con mayor atención por la biopolítica y la gubernamentalidad), una "verdad de sí mismo" (presente en la post-fenomenología) y una "verdad del objeto técnico" (que emerge, con mayor fuerza, en los nuevos materialismos). De hecho, la vinculación con la "verdad" es un asunto que enfatizan los propios analistas del movimiento $Q S$, al señalar que "datos como reveladores de la verdad sobre uno mismo" (KRISTENSEN; PRIGGE, 2018: 50; traducción propria) o "se cree que los datos que surgen de la cuantificación del cuerpo revelan algún tipo de "verdad objetiva" (...) se establece una relación directa entre el cuerpo y el sí mismo, 
entre la biología y el conocimiento, entre la tecnología y la verdad" (AJANA, 2017: 4; traducción propria).

En este sentido, el objetivo principal de este artículo será construir un dispositivo conceptual que permita distinguir de forma diferenciada estos tres juegos de verdad, a la par que conjugue, en un mismo plano, las diferencias que estos despliegan sobre el tablero de juego. Basándonos en los planteos foucaultianos sobre la cuestión de la verdad, sumando los postulados de Gilbert Simondon sobre el objeto técnico, propondremos un modelo tecnoaletúrgico que permita dar cuenta de los tres juegos de verdad anteriormente aludidos. Esto, lejos de constituirse en un fetiche teórico-conceptual, obedece a la necesidad de aprehender y comprehender la experimentación de sí mismos que ejecutan los $Q S$ al ensamblar, simultáneamente, verdades técnicas, disciplinares e individuales. Comencemos, por ende, caracterizando en líneas generales al movimiento $Q S$.

\section{¿Qué es el movimiento Quantified Self?}

El movimiento QS fue fundado el año 2007 en San Francisco por Gary Wolf y Kevin Kally, editores de la revista Wired. En el movimiento $Q S$ participan usuarios y fabricantes de herramientas de diferentes partes del mundo, con la finalidad de ejecutar experimentos de Self-tracking a través de la producción y análisis de datos numéricos. En este sentido, su principal slogan es "Self Knowlege Throught the Numbers". A partir de la creación de los QS labs se extienden rápidamente alrededor del globo, contando actualmente con 104 comunidades. Las distintas comunidades (que presentan diferentes grados de organización, implicación y actividad) realizan encuentros llamados Show and tell, lo cuales son convocados a través de la aplicación Meetup ${ }^{1}$. De igual modo, se realiza un gran encuentro anual que intercala su sede entre Europa y Estados Unidos.

El formato de dichos encuentros, a grandes rasgos, consiste en personas que exponen sus experimentos de cuantificación y comparten información relevante para la comunidad. Las exposiciones, en efecto, gravitan en torno a las siguientes preguntas: ¿qué hiciste? ¿cómo lo hiciste? ¿qué aprendiste? O bien, expresado en sus propios términos:

La comunidad del Quantified Self construye su conocimiento colectivo a partir de las personas que comparten las ideas extraídas de sus propios datos $n=1$. No sólo aprendemos de estos proyectos, sino que también nos inspiramos para llevar a cabo proyectos iguales o similares (TZOVARAS, 2018; traducción propria). 
En este sentido, un aspecto fundamental del movimiento es la reivindicación del propio cuerpo como espacio de investigación (a esto apunta, de hecho, la fórmla $n=1$ ), tanto en su calidad de investigadores y, simultáneamente, de objetos de investigación. Por ende, la novedad del movimiento $Q S$ no radica tanto en las prácticas de automonitoreo y cuantificación del cuerpo en la vida cotidiana, sino que, antes bien, en su articulación con las tecnologías de información y comunicación (TICs) y las tecnologías self-tracking, con la finalidad de digitalizar, producir y representar los datos del yo cuantificado de forma metódica y sistemática. Como manifiesta uno de sus fundadores:

pensamos en estas herramientas como si apuntaran hacia afuera, como ventanas, y me gustaría invitarles a pensar que también se vuelven hacia adentro y se convierten en espejos. De modo que cuando pensamos en utilizarlas para conseguir una mejora sistemática, también pensamos en cómo pueden ser útiles para la mejora personal, para el autodescubrimiento, la autoconciencia, el autoconocimiento (WOLF, 2010; traducción propria).

De esta manera, por medio de una serie de dispositivos inteligentes interconectados (wearables, smartphones, tablets, brazaletes, anillos o bio-sensores) que reportan y cuantifican en todo momento los signos vitales y las actividades cotidianas, los QSers se vuelven entidades híbridas o "cyborgs" mejorados. La compilación sistemática de datos, en efecto, busca la identificación de patrones de conducta (tanto a escala individual como en comparación con otros usuarios), que permitan la auto-comprensión, la transformación de sí mismos y el continuo mejoramiento. Entre las principales dimensiones de experimentación destaca la salud, el estado físico, el bienestar emocional, las relaciones sociales y la productividad en el trabajo. Los experimentos son variados y pueden incluir variables como horas de sueño, calorías quemadas e ingeridas, patrones de movimiento (trayectos recorridos o cantidad de pasos por unidad de tiempo), oxígeno en sangre, peso, etc. Otros, por su parte, realizan un monitoreo de patologías (como síntomas o efectos de un determinado tratamiento), la concentración de sustancias en el organismo, así como la monitorización de emociones y estados anímicos. Ruckenstein y Pantzar (2017) en un análisis de discurso sobre la revista Wired y el movimiento $Q S$, apuntan que los temas más relevantes son la transparencia, optimización, los circuitos de retroalimentación (feedback loops) el biohacking. Además, a esta multiplicidad de prácticas se conjuga con una participación igualmente plural y heterogénea, encontrando entre ellos diseñadores, científicos, 
académicos, empresarios, ingenieros, fabricantes y usuarios curiosos atraídos por este emergente campo de experimentación y estudio. Sin embargo, algunos autores (CHOE et al., 2014) han definido dos grandes perfiles al interior del movimiento: a) un grupo centrado en la dimensión del trabajo; y b) otro grupo vinculado principalmente al seguimiento de la condición de salud. Respecto a este último, en el cual los $Q S$ han tenido un fuerte impacto, se pueden mencionar como iniciciativas relevantes los experimentos de seguimiento de sintomatología y el desarrollo tecnologías (GIONES-VALLS; GIONES, 2015; SWAN, 2012), la organización del Cuarto Simposio de $Q S$ y Salud Pública celebrado en abril de 2018², así como la creación, en 2012, del Quantified Self Institute en la Universidad de Ciencias Aplicadas de Hanze (Groningen, Países Bajos), con la finalidad de investigar el uso de herramientas y métodos de auto seguimiento en materia de salud.

\section{Visiones críticas sobre el movimiento $Q S$}

\section{Estudios de Gubernanamentalidad y Biopolítica}

\section{a) La biopolítca de la cuantificación}

El concepto de Biopoder, originalmente planteado por Foucault (2002a), consistía en dar cuenta del proceso de politización que la vida adquiere a partir del siglo XVIII en Europa. De esta manera, la preocupación por la administración de la vida toma dos formas, enfocadas en objetivos distintos que se entrelazan: la anatomopolítica y la biopolítica. En primer lugar, la anatomopolítica o tecnologías disciplinarias abordan el cuerpo como máquina. Consiste en la supervisión constante, ininterrumpida de los procesos activos del cuerpo, procurando dividir, tanto como sea posible, en el tiempo y en el espacio, los movimientos del cuerpo con el objetivo principal de extraerle en profundidad un conocimiento y transformarlo simultáneamente en una máquina más potente y utilizable con determinados fines.

Los dispositivos disciplinares de fabricación de los cuerpos modernos han sido la escuela, la fábrica, la prisión y el manicomio. En este sentido, los dispositivos de monitorización y registro utilizados por la comunidad $Q S$, según Sanders (2017) son por excelencia los mecanismos disciplinarios contemporáneos. Estos facilitan el ejercicio de control en detalle y meticulosidad de las operaciones del cuerpo, simultáneamente cultivando capacidades y optimizaciones que convergen con las normas de la salud y la belleza socialmente establecidas. A diferencia de las formas modernas, ya no es necesario concentrar 
los cuerpos en un espacio determinado y serializar el tiempo, sino que el ejercicio de la anatomopolítica en la actualidad depende directamente de un individuo, que se monitoriza y mejora a sí mismo en cualquier momento y lugar gracias a los dispositivos móviles. Según Bruno (2013) la vigilancia contemporánea está distribuida y es inmanente, ya que los sujetos trasladan consigo, las tecnologías que los vigilan y convierten todas sus acciones en datos. En congruencia con esto Gordo López et al. (2013) sostienen que, el aspecto anatomopolítico de las prácticas $Q S$, se ve reflejado en la extracción de datos individuales como la posibilidad de optimizar y conocer el cuerpo humano para trascender sus límites biológicos y convertirse en máquinas más eficientes y productivas.

En segundo lugar, la biopolítica, que según Foucault (2002a) es una tecnología históricamente posterior y que necesariamente se incrusta sobre la anatomopolítica. Esta implica otra escala del ejercicio del poder y se disponen a intervenir sobre un cuerpo especie, es decir, sobre el conjunto de humanos como seres vivientes regidos por procesos biológicos. Se propone regular los procesos vitales de las poblaciones, como la reproducción, la mortalidad, la morbilidad a partir de cálculos estadísticos. Según Sanders (2017), la biopolítica contemporánea se ve enormemente potenciada, en la era digital por medio de la gestión del big-data. La capacidad de gestionar e interpretar la enorme cantidad de datos provenientes de los usuarios de dispositivos móviles y vestibles, que informan continuamente sobre hábitos cotidianos, alimentación, actividad física, patrones de movimiento; se vuelve una poderosa herramienta de producir conocimiento detallado de la conducta de la población por parte de los expertos y autoridades de la salud. A su vez, estos dispositivos y aplicaciones motivan a los usuarios a comprometerse con formas de vida saludables a través de monitorear diariamente sus actividades en función de indicadores de salud (alimentación, deporte, horas de sueño). De esta manera, según la autora el uso de dispositivos digitales de auto rastreo funciona como mecanismo biopolítico, ya que los cuerpos renderizados interiorizan las normas, encarnando hábitos y estilos de vida saludables, al mismo tiempo que se vuelven más visibles y más gestionables. Según Ajana (2017) asistimos a un cambio ontológico y epistemológico, en el cual nuestras vidas son tratadas como un proyecto de monitorización (selftraking proyect). La digitalización de casi todos los procesos del cuerpo mediante tecnologías biométricas se ha vuelto la principal forma de producción de saber y técnicas de conducción de la población de la biopolítica contemporánea (AJANA, 2012). 
Por otro lado, es interesante resaltar fenómenos vinculados a la biopolítica que no se encuentran asociados a la imposición de conductas normalizadas, sino más bien una cierta forma de gestionar y producir la vida que germina en los intersticios de las relaciones de poder. Nafus y Sharman (2014) consideran que existe un matiz en las prácticas de los $Q S$, que se escapa de la biopolítica regida por big data. Las autoras sugieren que los $Q S$ ejercen un soft-resistence al régimen de las empresas y la producción científica institucionalizada. Esto es posible ya que sus integrantes son capaces de tomar múltiples roles, como diseñadores de proyectos, recolectores de datos y creadores de sentido crítico. Esto cambia las formas habituales de registro de la información, el uso de los datos y sus prioridades, por lo que se vuelven menos capturables. Sin embargo, el termino soft sugiere que los modos de los $Q S$ no desafían el predominio del big data ni los constructos dominantes de la salud, sino que se encuentran en diálogo y en dependencia, ya que la mayoría utiliza los dispositivos en venta en el mercado. El movimiento $Q S$

no interactua con los algoritmos como incautos, ciegos o inconcientes, sino como participantes activos en un diálogo que se mueve entre los datos como una externalización del sí mismo y la comprensión interna, subjetiva y cualitativa de lo que significan los datos (NAFUS; SHERMAN, 2014: 1793; traducción propria)

Las prácticas $Q S$ no se agotan en la producción conocimiento sobre el yo, sino que mantienen prácticas en comunidad, donde se comparten datos y experiencias, que apuntan a lo que Lupton (2016a) llama saber colectivo (wisdom of crowd). Según Ajana (2017) estas prácticas colectivas $Q S$ pueden ser pensadas a partir del concepto sugerido por Rabinow (1996) de biosocialidad. Este concepto describe las modalidades de construir comunidades y asociaciones entre individuos a partir de rasgos biológicos compartidos (alteraciones en un cromosoma, portadores de VIH, pacientes con enfermedades raras). Estas comunidades activas, que en los últimos años han encontrado en la web un territorio propicio para su desarrollo, se apropian de narrativas científicas, se asocian con expertos en medicina y laboratorios, con el objetivo de producir nuevas técnicas alternativas de cuidado y tratamiento de sus vidas. Según Rose estos vectores son expresiones "desde abajo” que pluralizan la verdad biológica y biomédica, introducen dudas, controversia y reubican la ciencia en campos de la experiencia, la política y el capitalismo" (2012: 287). Sin enbargo, Harris, Kelly, y Wyatt (2015) presentan el concepto de autobiologías como contrapunto del concepto de biosocialidad, para entender las prácticas $Q S$. Las autoras utilizan el termino autobiologías 
para resaltar la importancia de las tecnologías móviles en la producción de narrativas individuales. El concepto que se desprende de biografía, implica la producción de una historia de sí incluyendo aspectos de la vida vinculados en la genética, la fisiología, lo molecular y lo celular. Las autoras sostienen que el uso de estas tecnologías con la que nos narramos, están transformando las formas en que nos comprendemos, inclusive hasta nuestra propia biología. El contrapunto con el concepto de biosociabilidad se sustenta en que en las autobiologías no se tratan de relatos de pacientes sino de individuos sanos. Además, si bien existen una gran cantidad de espacios en la web donde las personas comparten sus historias con otros, según las autoras, esto no constituye una identidad colectiva sino un individualismo colectivo.

\section{b) El ethos neoliberal y la gubernamentalidad algorítimica}

Esta línea se centra en los análisis sobre el liberalismo y neoliberalismo, no como una ideología ni como una escuela económica, sino como un arte de gobierno (FOUCAULT, 2006, 2007). Esto refiere puntualmente a una forma de dirigir la conducta de los individuos, creando un medio ambiente que necesariamente depende de la existencia de la libertad, en tanto una libre circulación de bienes y de libertades individuales. Esta racionalidad de gobierno tiene su mayor expresión contemporánea en la teoría del capital humano. Esta supone un sujeto activo, calculador y responsable capaz de incrementar su capital humano (cuerpo saludable, conocimiento, educación, etc), de tal manera que se dirige a sí mismo como una empresa, un empresario de sí. Esto implica que el capital deje de ser una categoría simplemente económica, dedicada a la circulación de bienes y a la acumulación; y pase a ser una categoría semiótica por la cual los individuos dan sentido a su existencia. De Sousa (2013) sostiene que las prácticas $Q S$ conforman el ideal de ciudadano neoliberal, ya que buscan optimizarse a partir de monitorearse voluntariamente, medirse y ejercitarse, tomando el control de sus cuerpos con la minuciosidad del detalle minuto a minuto. Esto se suma, a que dichas prácticas están asociadas a proyectos personales donde prima la cuestión del mejoramiento, el crecimiento y el auto desarrollo.

Por su parte Lupton (2014), sostiene que el movimiento $Q S$ es una expresión de la emergencia, de lo que llama Cultura Self-tracking. Esta implica la articulación de una serie de racionalidades, discursos, prácticas y tecnologías: a) una dinámica del conocimiento de sí 
mismo y de la conciencia de sí que se encuentra signada por un concepto del yo como empresario de sí mismo; b) la existencia de una serie de moralidades y políticas que suponen un sujeto como actor individual y racional responsable por su propia vida y bienestar; c) las tecnologías digitales han incrementado su capacidad para monitorear aspectos del cuerpo humano, comportamientos, hábitos y medio ambiente; d) la emergencia de un saber económico sobre los datos, para el cual, tanto los datos individuales de un usuario como los flujos masivos de datos son vistos como mercancías comerciables; e) la comprensión de parte de los gobiernos, la gestión, actores comerciales y agencias que los datos derivados del auto monitoreo pueden ser dirigidos a sus propios propósitos. Según Lupton "el valor que se atribuye a los conjuntos de datos digitales personales combina dos formas de valor: el relacionado con la economía de los datos digitales y el que surge de la capitalización del cuerpo humano" (2014: 14; traducción propria). Dentro de este entramado neoliberal, Whitson (2013) destaca las técnicas de gamification como una forma de auto-vigilancia y motivación para promover cambios en el comportamiento o adquirir conductas nuevas. La gamificación combina, por un lado, el diseño lúdico para motivar a los usuarios a competir entre ellos en retos para alcanzar metas y premios; y por otro, los perfiles de usuario de las redes sociales (facebook, Twitter, linkedln). De esta manera, promueve una subjetividad lúdica donde los usuarios exponen voluntariamente información personal. Esta gran acumulación de datos es simplificada y representada en barras de progreso, cuadros y gráficos fácilmente comprensibles por los usuarios.

Otros estudios centran su foco de interés en la producción y gestión de los datos, como uno de los mecanismos más relevantes de conducción de la conducta de los individuos en las sociedades contemporáneas. Se sugiere que el desarrollo tecnológico y sus nuevas capacidades de almacenar y gestionar flujos de información cada vez más voluminosos, produce un proceso de naturalización de los datos en la vida cotidiana. En este sentido, Reigeluth (2014) plantea que casi todas las actividades producen rastros digitales que pueden ser almacenados, ordenados y utilizados, donde devenimos en una colección de rastros digitales. Nos convertimos en datos, somos construidos en identidades algorítmicas (CHENEY-LIPPOLD, 2011). En congruencia con esto, el concepto de gubernamentalidad algorítmica (ROUVROY; BERNS, 2015) intenta dar cuenta de esta nueva racionalidad, que se sostiene en la recolección, agrupación y análisis automatizados de grandes cantidades de 
datos para modelizar, anticipar y afectar los comportamientos de los individuos. Los autores describen el procedimiento en tres momentos: i) almacenamiento de datos: una gran cantidad de datos son sistemáticamente recolectados de las prácticas y de las interacciones; ii) minería de datos: proceso posterior donde se extraen correlaciones y patrones a través de algoritmos de cálculo; y iii) perfilado: no se trata tanto de identificar a una persona específica sino correlacionar ocurrencias dispersas, juntarlas para reducirlas a comportamientos.

\section{Postphenomenological Studies}

Desde esta línea de investigación, a diferencia de la anterior, el protagonismo del análisis corre por cuenta del sujeto individual y la consciencia. Si bien la fenomenología de Husserl, sobre la que estas corrientes se asientan, no ignora los factores intersubjetivos, históricos y sociales, resulta evidente que el énfasis se encuentra puesto en cómo el mundo emerge para una consciencia. En efecto, Husserl (1962: 136-137) sostendrá enfáticamente que "esta es la norma que queremos seguir como fenomenólogos: no tomar en cuenta nada más que aquello que en la conciencia misma, en pura inmanencia, podamos ver con evidencia esencial". No obstante, la modalidad eminentemente intelectualista con que Husserl desarrolla su trabajo, hará que surja una primera corriente de post-fenomenólogos franceses (Merleau-Ponty, Lévinas, Henry) en que la corporalidad jugará un rol fundamental. Clarificante, en este sentido, resulta el siguiente fragmento de Lévinas (2002: 148): "el cuerpo es una permanente puesta en duda del privilegio que se atribuye a la conciencia de «dar el sentido» a todo".

Siguiendo esta línea, pero incorporando además la relación con artefactos tecnológicos, es que surgirá una segunda camada de estudios post-fenomenológicos. Ihde (1979) inaugura una filosofía de la tecnología tomando esta tradición de pensamiento centrada en la ralación humano-tecnología. Su pregunta central está vincualada a qué rol juegan los artefáctos técnicos en la interacción entre humanos y mundo, así como la constitución de la subjetividad y la objetividad en este proceso. Verbeek (2005, 2016) sostiene que el enfoque postfenomenológico sugiere una teoría de la mediación tecnológica, donde los artefactos tecnológico no son meros intrumentos funcionales, sino que participan en la co-formación del mundo. Estos estudios son fundamentalmente empíricos y centran su 
analisis filosófico en las tecnologías actuales, por lo que hacen una filosofía desde la tecnología.

Ahora bien, en lo que al movimiento $Q S$ se refiere, el trabajo de Kristensen y Ruckenstein (2018), propone comprender las prácticas de monitorización del movimiento QS en el marco de un "laboratorio del yo", donde las tecnologías funcionan como recursos que colaboran en el self-macking y el self-improvement. Esta perspectiva se enfoca en entender cómo los usuarios experimentan el data self y como las prácticas de self-tracking se ven implicadas a procesos de restructuración de la experiencia corporal. En este sentido, sin alejarse de un enfoque crítico, plantean que las tecnologías de monitorización se convierten en participantes de la experiencia de sí mismo, donde algunos aspectos del yo son amplificados mientras otros son reducidos o restringidos. En un trabajo similar, Kristensen y Prigge (2018) proponen una tipología de las asociaciones entre tecnología y sí mismo en las prácticas de self-tracking. Esta tipología se compone de cuatro aspectos; i) Una Hermenéutica del Self/Enactment: mientras que el concepto de hermeneutica refiere a la interpretación, la representación y la comprensión, según las autoras en el self-tracking también esta asociado a una forma de conocerse y actuar sobre sí mediada por los datos. La producción de datos alienta a los usuarios a persibir un yo corporal através de evaluaciones e interpretaciones de datos. La hermeneutica del self/enactment refeiere a un yo de los datos (data double), como una forma de saber que puede ser reflexionada y utilizada para varios propositos. ii) Una encarnmación del Self/Experience: este punto se ha visto favorecido gracias a la reducción del tamaño de los dispositivos y sus diseños vestibles. Ilhe ha llamado este fenómeno como relaciones corporizadas (embodiment relations) y que Verbeek las ha pensado como relaciones Cyborg. La idea de Cyborg implica que lo humano y la tecnología forman una nueva entidad o una nueva experiencia del cuerpo donde el sujeto siente que el dispositivo forma parte o es una extensión de su cuerpo. A este modo de relación, las autoras lo llamam experience, ya que entienden que esta nueva entidad, compuesta de carne y circuitos electromagnéticos es la que experimenta (new experiencing entity). En este sentido, las tecnologías son vividas e integradas a la experiencia, otorgando otra sesibilidad y otros atribututos sensoriales, no solo en lo concerniente a la auto percepción, sino también en las formas de percibir el mundo. En este aspecto, Van Den Eede (2015) propone el concepto de enmeshment, que puede ser traducido como engranaje, para comprender las relaciones 
particulares que las tecnologías self-traciking implican entre cuerpo, tecnología y la perseptual experience. iii) Alterity/Entanglement: este aspecto refiere a cómo el usuario toma conciencia de cómo la tecnología amplifica, reduce o incluso contradice la experiencia subjetiva del yo. A diferencia de los dos modos de asosociación anteriores, donde la tecnología se percibe como un continuom del yo, en este modo es percibida como alteridad. Así, le llaman entanglement al resultado de la negociación entre los usuarios y las tecnologías de datos. iiii) Background/Integration: este último punto refiere a la integración de la tecnología de forma activa en la vida de los usuarios, es decir que el sujeto pasa por un proceso de evaluación del dispositivo a partir del uso prolongado y cotidiano para luego integrarlo como una especie de background. Según las autoras, este último aspecto es el que distingue a los sujetos de movimiento $Q S$ con el resto de usuarios que simplemente utilizan tecnologías de self-tracking.

\section{La emergencia de los Nuevos materialismos}

La preocupación teórica y epistemológica por la materialidad concreta, como opuesta a la idealidad abstracta, es una disputa que se remonta a los inicios de la filosofía griega. No será, sino hasta la época moderna que este concepto será popularizado por el uso despectivo con que los filósofos de la Escuela de Cambridge (Cudworth, Culverwel, More) se enfrentan a lo que denominan como el materialismo de Hobbes. Posteriormente, en un contexto de crítica a las corrientes principales del idealismo alemán, las elaboraciones de Marx (así como las de Schopenhauer y Nietzsche) enfatizarán la importancia de las condiciones materaliales de la existencia individual concreta frente a la defensa de conceptos universales abstractos que sostenían las filosofías de Kant, Fichte y Hegel. El materialismo dialéctico, en este sentido, ejercerá una enorme influencia en el contexto social, histórico y político del siglo $\mathrm{XX}$, dando pie a constantes relecturas y reinterpretaciones (como el materialismo cutural de Harris o la refundación del mismo ejecutada por Žižek desde una coordenada lacanianohegeliana).

Por otra parte, en la actualidad asistimos a un verdadero auge en la emergencia de enfoques semiótico-materiales (LATOUR, 2008) y de nuevos materialismos (LEMKE, 2015; PFEIFER, 2015). El realismo agencial de Karen Barad (2007), por ejemplo, sostiene que la 
materia es una substancia que en su devenir intra-activo no se constituye en una cosa, sino en un hacer, en una congelación de la agencia:

la noción de intraacciones reformula las nociones tradicionales de causalidad y agencia en una reconfiguración continua tanto de lo real como de lo posible. En particular, la agencia se desprende de su órbita humanista tradicional. La agencia no está alineada con la intencionalidad o la subjetividad humana (BARAD, 2007: 234-235; traducción propria).

En este sentido, para el nuevo materialismo dejan de ser pertinentes las distinciones y dicotomías clásicas que separan al animal de lo humano, así como a lo natural de lo tecnologico. El objeto técnico, por ende, no se escapa a los intereses que ponen sobre la mesa los nuevos materialismos.

Los últimos trabajos de Lupton $(2016 b, 2017)$ apelan a los planteos sociomaterialistas contemporáneos para construir un modelo analítico sobre los personal digital data assemblages y su relación con el yo y el cuerpo. Señala que las perspectivas del materialismo feminista, el materialismo vital y la antropología de la cultura material, aportan conceptos relevantes para describir la forma que estos ensamblages (datos-cuerpos-selves) operan, al mismo tiempo que se hacen, crecen, se articulan y se entanglement con otras entidades humanas y no-humanas. El enfoque difractivo que propone Lupton sugiere tener en cuenta las siguientes posiciones ontoepistemológicas: primero, reconocer que tanto humanos como no-humanos estan inbricados en formas híbridas, inestables y generativas, de tal manera que estos no pueden ser comprendidos autonomamente. Segundo, y en resonancia con lo anterior, la agencia y las capacidades vitales se encuentra distribuidas entre entidades diversas en ensamblages humanos/no-humanos. Tercero, el significado que los usuarios hacen de los datos es de naturaleza sensible, corporizada y materializada, que funda una forma de conocer, percibir y hacer el cuerpo y la vida. Cuarto, los significados de los datos son cambiantes a medida que se mueven en diferentes tipos de ensamblages. Y quinto, una perspectiva que permita rastrear las formas en que humanos y no-humanos se enrriedan, identificando así prácticas, enacciones y efectos en las vidas humanas.

\section{Tecno-aleturgia: un modo de articular los juegos de verdad}

Como hemos revisado, las diversas líneas de investigación sobre los $Q S$ centran su atención en diferentes dimensiones de análisis: lo colectivo, lo individual y lo técnico. Inspirándonos en los trabajo de Foucault y Simondon, sostendremos que estas dimensiones 
se corresponden con el despliegue de tres juegos de verdad: disciplinar, aletúrgico y técnico. La preocupación por la "verdad", incluso, ya se encuentra presente en las líneas de investigación precedentes, cuando se menciona a los "datos como reveladores de la verdad sobre uno mismo" (KRISTENSEN; PRIGGE, 2018: 50; traducción propria) o "se cree que los datos que surgen de la cuantificación corporal revelan algún tipo de "verdad objetiva" (...) establece una relación directa entre el cuerpo y el sí mismo, entre la biología y el conocimiento, entre la tecnología y la verdad" (AJANA, 2017; traducción propria). Foucault, por su parte, es enfático en recalcar que "el poder en general, no podría ejercerse si la veardad no se manifestara" (2014: 93).

En este sentido, nuestro modelo conceptual, llamado Tecno-aleturgia, será definido como el agenciamiento de tres juegos o modos de verdad: a) en primer lugar, una Verdad disciplinar que, en la teorización de Foucault, se correspondería con los denominados dispositivos de saber-poder y el ejercicio de "estar en la verdad"; b) la dimensión de la Verdad aletúrgica, la cual se corresponde con lo que Foucault, en sus cursos de la década de 1980 en el Collège de France, denomida como práctias aletúrgicas y la puesta en ejercicio de un "decir veraz"; y c) por último, la inevitable presencia de una Verdad técnica; es decir, el necesario ensamblaje con objetos técnicos que requiere el despliegue de la verdad disciplinar y aletúrgica, lo cual implica el reconocimiento de una verdad propia e inherente al modo de existencia del objeto técnico.

Si bien esta última dimensión resulta ajena a la problematización de Foucault, lo que nos interesa primeramente destacar es la importancia que este otorga al problema de los juegos de verdad y los régimenes de verdad. Así, centraremos la atención en la articulación que Foucault realiza, con mayor claridad hacia el final de su vida, entre los conceptos de verdad y sujeto. Dicho en sus propias palabras: "mi trabajo ha tratado de tres modos de objetivación que transforman a los seres humanos en sujetos" (FOUCAULT, 2015a: 318). El primer modo de objetivación resulta de las prácticas que se consideran a sí mismas como científicas, las cuales podemos ordenar en la obetivación del sujeto hablante (gramatica general, filología, lingüística), el sujeto productivo (análisis de la riqueza, economía política) y el sujeto vivo (historia natural, biología) (FOUCAULT, 2002b). El segundo modo, se vincula con lo que Foucault llamó las prácticas divisorias, aquellas que establecen distinciones, nominaciones y tratamientos de exclusión. Estas prácticas establecen una 
división entre lo normal y lo patológico, lo sano y lo enfermo, el loco y el cuerdo, el peligroso y el buen ciudadano (FOUCAULT, 1976, 2015b; 2015c). Por último, el tercer modo de objetivación lo constituyen las prácticas de sí por las cuales los sujetos pueden ejercer sobre sí mismos técnicas para comprenderse, transformarse o mejorarse a sí mismos (FOUCAULT, 2012a; 2012b). Ahora bien, cabe destacar que con anterioridad al estudio de las técnicas de sí, el trabajo foucaultiano estaba centrada en el problema de las redes de saber-poder. Esto implicaba pensar al sujeto como un efecto de unas relaciones de fuerzas y unas formas de conocimiento; es decir, que la subjetividad podría ser comprendida como la propiedad emergente de un dispositivo o de una episteme. En este sentido, Foucault comenzará a reformular este esquema al plantear una analítica de la gubernamentalidad, donde el poder es comprendido en términos de gobierno. Este, por ende, debe entenderse no como una instancia suprema encargada de las desiciones ejecutivas y administrativas de un Estado, sino como los mecanismos y los procedimientos dedicados a conducir la conducta de sí mismo o de otros (FOUCAULT, 2006, 2007). De este modo, se planteará el estudio de los régimenes de verdad, preguntándose en qué condiciones históricas y con qué efectos es posible el ejercicio de una veredicción, distinguiendo entre estructuras epistemológicas y formas aletúrgicas (FOUCAULT, 2010, 2014).

En lo concerniente al estudio de las estructuras epistemológicas, lo fundamental pasa por analizar cómo se produce el conocimiento verdadero. Además, se debe indagar en cómo estos discursos establecen límites y normas, organizandose en disciplinas, de tal manera que las prácticas de enunciación están en la verdad o fuera de esta. Esta distinción de las estructuras epistemológicas se correponde con lo que nosostros hemos denominado como verdad disciplinar. Por otro lado, las formas aletúrgicas (es decir, lo que en nuestro modelo conceptual corresponde con la verdad aletúrgica) implican un cierto uso ético de los discursos, en tanto que el sujeto hace pasar en su relación consigo mismo y con los otros un cierto decir veraz, un ejercicio que le posibilita enunciar la verdad sobre sí mismo. En este sentido, según Foucault, habría una especie de pragmática de la verdad en el sujeto que no puede ser reducida a las estructuras epistemológicas del conocimiento. Por ello, con este concepto Foucault reformulará su propuesta de saber-poder, para plantear la noción de "gobierno de los hombres por la verdad" (FOUCAULT, 2014: 30). El saber ya no es solamente aquello que necesariamente debe ser producido para gobernar, una bateria de 
conocimientos construidos objetivamente, verificables y utilitarios, sino una manifestación de la verdad que hace posible el ejercicio de poder. De este modo, la relevancia pasa a ser de los régimenes de verdad y de cómo el sujeto, a través de una serie de actos, puede decir la verdad sobre sí mismo. En concreto, Foucault nos hablará de las formas aletúrgicas, entendidas como un régimen definido por la obligacion individual de establecer consigo mismo una relacion de conocimiento permanente que permite descubrir los secretos que, en el fondo de sí mismo, se esconden y se escapan. Se trata, en definitiva, de la obligación "de manifestar esas verdades secretas e individuales a través de actos que tienen efectos, efectos especificos mucho más allá de los efectos de conocimiento: efectos liberadores" (FOUCAULT, 2014: 107). De esta manera, quedan definidos los dos primeros juegos de verdad que constituyen nuestro modelo tecno-aletúrgico. Como señala Foucault:

se puede decir la verdad siempre que se diga en el espacio de una exterioridad salvaje; pero no se está en la verdad más que obedeciendo a las reglas de una "policía» discursiva que se debe reactivar en cada uno de sus discursos (FOUCAULT, 1992: 22).

Ahora bien, a la "verdad disciplinar" (y sus modos de estar en la verdad) y la "verdad aletúrgica" (y sus modos de decir la verdad), debemos conjugar un tercer nivel de veridicción que configura aquello que hemos denominado como tecno-aletúrgia. Nos referimos, específicamente, al nivel de la "verdad técnica" que es puesta en juego por el modo de existencia de los objetos técnicos. Si bien Foucault no plantea directamente la cuestión de la verdad en el objeto, no es menos cierto que, en su curso de 1980, parece rozar y acercarse a este importante asunto, el cual ya había sido trabajado por autores franceses de la época como André Leroi-Gourhan, Gilbert Simondon y, posteriormente, Bernard Stiegler. Menciona

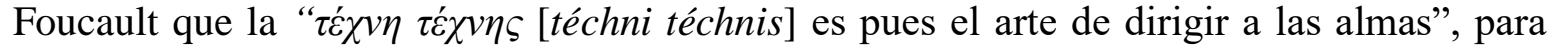
insistir -inmediatamente-, en que "lo que querría hacer este año es, precisamente, estudiar la relación entre la $\tau \varepsilon ́ \chi v \eta \tau \varepsilon ́ \chi v \eta \varsigma$ [téchni téchnis] como arte supremo, vale decir, arte de gobernar a los hombres, y la aletúrgia" (2014: 71). Dicho en otros términos: Foucault remarca la importancia del vínculo existente entre la técnica y las manifestaciones de verdad propias de la aletúrgia.

Sin embargo, la conceptualización foucaultiana de la técnica se centra exclusivamente en dos grandes áreas (técnicas de gobierno y técnicas de sí), dejando sin teorizar la posible existencia de un régimen de verdad concerniente al propio objeto técnico. Este, en efecto, 
será uno de los problemas fundamentales de la obra de Simondon, en la cual se busca "suscitar una toma de consciencia del sentido de los objetos técnicos [...en tanto que] mediadores entre la naturaleza y el hombre" (2007: 31). Para este enfoque, el objeto técnico se define en sí mismo, teniendo una evolución propia que pasa por tres niveles: el elemento técnico (las piezas/herramientas), el individuo técnico (las máquinas) y el conjunto técnico (las redes). En este sentido, el objeto técnico tiene un modo de existencia propio ${ }^{3}$ (lo aquí entendemos como "verdad técnica"), que sirve de mediador entre los juegos de verdad disciplinares y aletúrgicos.

Para Simondon, de hecho, la toma de consciencia de la verdadera naturaleza de las máquinas ${ }^{4}$ resulta fundamental para conseguir una renovada concepción de la cultura que permita sintetizar la ruptura y desdoblamiento de las tendencias teóricas y prácticas que reproducen dicotomías tales como sujeto-objeto, natural-artificial individual-social. En nuestros términos, esto equivaldría a la consecución de una articulación entre la dimensión disciplinar de estar en la verdad y la dimensión aletúrgica del decir veraz. En esta línea, la cultura se constituye en un centro neutro (a saber, en una red técnica) en que se resuelve el problema de la articulación entre lo individual y lo social. No es, por ende, en el nivel de la individuo/sujeto (como pensaba Foucault) en donde el problema de lo social se desarrolla y resuelve. Antes bien, para Simondon, el verdadero nivel de individuación de la realidad humana es la cultura; pero, como hemos insistido, una red cultural que toma consciencia del rol desempeñado por el objeto técnico. Y esta toma de consciencia, para ser completa, "debe desarrollar la red de puntos relacionales entre el hombre y el mundo, convirtiéndose en tecnología, es decir, una técnica de segundo grado que se ocupa de organizar estos puntos relacionales" (SIMONDON, 2007: 243).

Por ende, cuando la cultura deja de desconfiar de la técnica, "el ser humano es puesto realmente en situación" de resolver el problema; es decir: va más allá de lo individual y de lo meramente conceptual para ingresar en un campo experiencial. Ser experimentos, y tener el valor de serlo, apunta precisamente a esto: "lo que debe ser experimentado es la puesta en situación particular en la red técnica, en la medida en que coloca al hombre en presencia y en el interior de una serie de acciones y procesos que él no dirige en soledad, sino en los cuales participa" (SIMONDON, 2007: 245). En este sentido, el movimiento QS se constituye en un grupo privilegiado para analizar este proceso. En tanto que articulan simultáneamente 
verdades disciplinares (por ejemplo, de la ciencia o la biomedicina), verdades técnicas (de los dispositivos tecnológicos utilizados para el self-tracking) y verdades aletúrgicas (que ponen en juego la subjetividad, una verdad sobre sí), el movimiento $Q S$ nos permite hacer la experiencia concreta de una tecno-aletúrgia fundamental para la sociedad de nuestros días.

De este modo, deberíamos enfatizar al menos dos aspectos en donde el modelo Tecnoaleturgico es algo más abarcativo para pensar el fenómeno contemporáneo del movimiento QS. En primer lugar, el planteo de Foucault (2002a) sobre el biopoder permite pensar cómo la vida se coloca en el centro de la política, a partir de una serie de transformaciones técnicas que se van a diferenciar de los modos de soberanía del ejercicio del poder. Esto se sostiene fundamentalmente en una voluntad de saber acerca de los procesos de la vida y sus cirscunstancias. Emergen así, disciplinas como la anatomomía, la biología, la economía política, la estadística entre otras ciencias humanas. Estas formas de saber se han desarrollado conjuntamente con las técnicas de conducción (anatomopolíca, biopolítica) que definen un cuerpo-máquina y un cuerpo-especie. En efecto, esta voluntad de saber es a la vez, como bien lo demuestra Foucault, una voluntad de poder, o dicho de otro modo, para conducir la vida es necesario una producción de conocimiento que la convierta en un objeto inteligible y operable. Del mismo modo, Rose (2012) quien retoma el concepto de biopolítica para analizar una serie de mutaciones contemporáneas, desatadas en parte, por nuevas técnicas de visualización del cuerpo somático y la incidencia de nuevas diciplinas (genética, biología molecular), sugiere que la emergencia de prácticas biomédicas consteladas entre el laboratorio, la clínica y el mercado, han estado transformando las propias formas de conducir y conceptualizar la vida. Tanto para Foucault como para Rose, quienes han convertido sus análisis en un punto de paso obligado para pensar los procesos de politización de la vida, ubican el despliegue de la biopolítica privilegiando las formas de producción de verdad disciplinares, es decir a partir de una serie de estructuras espistemológicas, dejando de lado las dimensiones propuestas por la tecno-aleturgia como la verdad aletúrgica y la verdad técnica. En segundo lugar, a diferencia de una visión biopolítica y gubernamental en donde los objetos técnicos tienden a ser vistos como dispositivos constrictivos y fundamentalmente disciplinares (recordemos, por ejemplo, la gubernamentalidad algorítmica), el modelo tecnoaletúrgico posibilita la comprensión del objeto no sólo como mediador (al estilo de la Teoría del Actor-Red), sino que también como amplificador de los juegos de verdad 
desplegados por un sujeto. En efecto, como bien apunta Simondon, no es en la individuación psíquica del sujeto en que se debe resolver la problematicidad inherente del campo psicosocial, sino que en un proceso de individuación colectiva en que el objeto técnico se constituye en un pluggins que nos conecta a la red y, además, pone en juego una verdad y un modo de existencia propio. Un $Q S$ r que, por ejemplo, diseña una herramienta tecnológica que le permite cuantificar alguna actividad cotidiana, no sólo se conecta a una red mediante la producción y subida de datos que son socializados en una plataforma, sino que se conecta a la verdad autónoma de un objeto que, al ser ejercitada y ejecutada por sí mismo, amplifica su propia verdad en tanto que sujeto. Los distintos tipos de verdad (disciplinar, aletúrgica y técnica) son autorecursivos y autónomos, pero resuenan los unos en los otros. Y, esta resonancia, se transmite y amplifica mediante una operación activa.

De este modo, a pesar de que el proceso aletúrgico no se resuelva en la verdad del sujeto (como, de diferentes modos, plantean los estudios postfenomenológicos y postfoucaultianos), esto no implica eliminar y/o silenciar al sujeto en el despliegue de esta partida de juego. Ser experimentos, y tener el valor de serlo, implica lanzar indefectiblemente los tres dados que nos han sido dados. Implica amplificar un decir veraz no sólo indagando en los secretos que guarda y esconde un sí mismo, sino que explorando el estar en la verdad disciplinar y la verdad tecnológica inherente a las redes de objetos. Así, la autonomía autorecursiva de cada juego de verdad se amplifica y resuena en la red, sin caer en una homogenización simétrica (al estilo de laTeoría del Actor-Red) o en la lógica del punto/dimensión privilegiado (como ocurre con las diferentes líneas de investigación sobre los $Q S$ que hemos revisado). No se trata, por ende, de conectores y mediadores que ejercen traducciones, sino de dispositivos que, poniendo en juego modos de verdad diferenciados, conectan dimensiones heterogeneas sin inyectar trascendencia y sin fracturar la red tecnoaleturgica que opera como un plano.

\section{Conclusiones}

En este artículo hemos expuesto los trabajos académicos que se encuentran, a nuestro entender, dentro de las perspectivas críticas acerca del movimiento QS. Estos estudios se han concentrado en diversos aspectos sumamente relevantes en la cuestión de las prácticas selftracking de los $Q S$, pero han dejado de lado el problema de la verdad. Pero, ¿por qué es 
relevante la cuestión de la verdad? Primero, porque para la propia comunidad $Q S$ se constituye en una dimensión importante (recordemos, por ejemplo, la idea del n=1 que, en su experimentación, nos entrega una verdad del sí mismo). Segundo, porque la propia teorización de Foucault termina por concebir a la verdad como un factor fundamental no sólo de las prácticas de sí mismo, sino también para el funcionamiento de los dispositivos de saber-poder. Tercero, porque reconocer que el objeto técnico tiene un modo de existencia propio implica asumir su irreductible autonomía, la cual no puede ser reducida a la verdad aleturgica y/o a la verdad disciplinar.

Ahora bien, ¿cómo se articula la autonomía de los tres juegos de verdad que implica la ejercitación de la tecno-aleturgia? En primer lugar, asumiendo que si bien el modelo tecnoaletúrgico se ensambla como una red, esto no quiere decir que los tres juegos se disuelvan en ella. Antes bien, cada juego mantiene su radical autonomía y su modo diferenciado de existencia. En segundo lugar, aprehendiendo que la tecnoaletugia es un proceso de amplificación resonante que comunica sin traducir y que conecta sin homogeneizar. Enfocarse, de este modo, sólo en hacer vibrar una de las dimensiones (por ejemplo, el puro decir veraz de mi propio cuerpo; o el puro estar en la verdad con que nos disciplina un cuerpo médico), dificilmente permita la ejecución de un amplificación resonante. Sin embargo, incluso la posición de sujeto, así como el saber-poder disciplinario, deben ser puestos activamente en juego. En tercer lugar, se debe comprenhender que la amplificación resonante se ejecuta como una experimentación (de lo disciplinar, de lo aleturgico y de lo técnico). Y, experimentar cada uno de estos juegos, implica estar dispuesto a jugar su juego. Así, no basta con utilizar cotidiamente una infinidad de dispotitivos tecnológicos para jugar el juego de lo técnico, así como vivir cada día en el mismo cuerpo no asegura alcanzar la sabiduría, la felicidad o el autoconocimiento. Por último, "ser experimentos y tener el valor de serlo", implica precisamente una ejecución activa y simultánemanete de las tres dimensiones. En este sentido, resultaría de gran relevancia aplicar empíricamente este modelo al movimiento $Q S$, y así explorar experimentalmente si nuestro juego conceptual resulta nutritivo y, si el de ellos, pone en juego algo más que la cuantificación del sí mismo.

\section{Referencias}

AJANA, Btihaj. Biometric citizenship. Citizenship Studies, vol. 16, nº 7, p. 851-870, 2012. 
AJANA, Btihaj. Digital health and the biopolitics of the Quantified Self. Digital Health, vol. 3, p. 1-18, 2017.

BARAD, Karen. Posthumanist Performativity: Toward an Understanding of How Matter Comes to Matter. Signs: Journal of Women in Culture and Society, vol. 28, no 3, p. 801-831, 2003.

BARAD, Karen. Meeting the universe halfway: quantum physics and the entanglement of matter and meaning. Durham: Duke University Press, 2007.

BARRETT, Estelle; BOLT, Barbara. Carnal knowledge: towards a 'New Materialism' through the arts. Em: COOLE, Diana.; FROST, Samantha. (Eds.). New Materialisms: Ontology, Agency, and Politics. Durham/London: Duke University Press, 2010. .

BEER, David. Governing through biometrics: the biopolitics of identity. Information, Communication \& Society, vol. 17, nº 8, p. 1051-1054, 2014.

BRUNO, Fernanda. Máquinas de ver, modos de ser: vigilância, tecnologia e subjetividade. Porto Alegre: Sulina, 2013.

CHENEY-LIPPOLD, John. A New Algorithmic Identity: Soft Biopolitics and the Modulation of Control. Theory, Culture \& Society, vol. 28, nº 6, p. 164-181, 2011.

CHOE, Eun Kyoung; LEE, Nicole; LEE, Bongshin.; PRATT, Wanda Marie; KIENTZ, Julie. Understanding quantified-selfers' practices in collecting and exploring personal data. ACM Press, 2014. Disponível em: <http://dl.acm.org/citation.cfm?doid=2556288.2557372>. Acesso em: 30 mar. 2017

CROCKETT, Clayton.; ROBBINS, Jeffrey. Religion, politics, and the earth: the new materialism. S.1.: Palgrave Macmillan, 2015.

DE SOUZA, Poppy. Self-tracking and body hacking: the biopolitics of the Quantified Self in the age of neoliberalism. The Transfigure Project, 2013. Disponível em: <https://bodycartography.wordpress.com/2013/06/11/self-tracking-and-bodyhacking-the-biopolitics-of-the-quantified-self-in-the-age-of-neoliberalism/>. Acesso em: 16 abr. 2018

FOUCAULT, Michel. Vigilar y castigar: nacimiento de la prisión. Mexico: Siglo Veintiuno Editores, 1976.

FOUCAULT, Michel. El orden del discurso. Buenos Aires: Tusquets, 1992.

FOUCAULT, Michel. Historia de la sexualidad: la voluntad de saber. Buenos Aires: Siglo XXI, 2002a. 
FOUCAULT, Michel. Las palabras y las cosas: una arqueologia de las ciencias humanas. Buenos Aires: Siglo XXI Ediciones, 2002b.

FOUCAULT, Michel. Seguridad, territorio, población: curso en el Collége de France (19771978). Buenos Aires: Fondo de Cultura Económica, 2006.

FOUCAULT, Michel. Nacimiento de la biopolítica: curso en el Collége de France (19781979). Buenos Aires: Fondo de Cultura Económica, 2007.

FOUCAULT, Michel. El coraje de la verdad: el gobierno de sí y de los otros II. Curso en el Collège de france (1983-1984). Buenos Aires: Fondo de Cultura Económica, 2010.

FOUCAULT, Michel. Historia de la sexualidad: el uso de los placeres. Madrid, España: Siglo XXI, 2012a.

FOUCAULT, Michel. Historia de la sexualidad: la inquietud de sí. Madrid, España: Siglo Veintiuno Editores, 2012b.

FOUCAULT, Michel. Del gobierno de los vivos: curso en el Collège de France (1979-1980). Buenos Aires, Argentina: Fondo de Cultura Económica, 2014.

FOUCAULT, Michel. La ética del pensamiento: para una crítica de lo que somos. Madrid: Biblioteca Nueva, 2015a.

FOUCAULT, Michel. Historia de la locura en la época clásica I. México D.F.: Fondo de Cultura Económica, 2015b.

FOUCAULT, Michel. Historia de la locura en la época clásica II. México, D.F.: Fondo de Cultura Económica, 2015c.

GIONES-VALLS, Aina.; GIONES, Ferran. Quantificar-se per viure a través de les dades: les dades massives (big data) aplicades a l'àmbit personal. BiD: textos universitaris de biblioteconomia i documentació, no 34, 2015.

GORDO LÓPEZ, Ángel.; RIVERA, Javier de; LÓPEZ LOSADA, Yago. Sociogénesis de las nuevas enfermedades tecnológicas y los dispositivos de auto-cuantificación. Quaderns de psicologia, vol. 15, nº 1, p.81-93, 2013.

TZOVARAS, Bastian Greshake. Share your method of analysis without sharing your data Quantified Self. Disponível em: <http://quantifiedself.com/2018/05/open-humanspersonal-data-notebooks/>. Acesso em: 18 maio. 2018.

HARAWAY, Donna. Manifiesto ciborg: el sueño irónico de un lenguaje común para las mujeres en el circuito integrado. Trad. Manuel Talens. California, 1991. 
HARRIS, Anna.; KELLY, Susan; WYATT, Sally. Autobiologies: Making sense of engagements with healthcare technologies. Eä - Revista en Salud, Sociedad, Ciencia y Tecnología, vol. 7, $\mathrm{n}^{\mathrm{0}}$ 2, p. 71-86, 2015.

HUSSERL, Edmund. Ideas relativas a una fenomenología pura y una filosofía fenomenológica. Mexiko: Fondo de Cultura Económica, 1962.

IHDE, Don. Technics and Praxis: A Philosophy of Technology. [s.1.] Springer Netherlands, 1979.

KRISTENSEN, Dorthe Brogaard; PRIGGE, Carolin. Human/Technology Associations in Self-Tracking Practices. Em: AJANA, Btihaj. (Ed.). Digital health and the biopolitics of the Quantified Self. London: Springer International Publishing, 2018. p. 43-59.

KRISTENSEN, Dorthe Brogard; RUCKENSTEIN, Minna. Co-evolving with self-tracking technologies. New Media \& Society, vol. 20, n 10, p. 3624-3640, 2018.

LATOUR, Bruno. Reensamblar lo social: una introducción a la teoría del actor-red. Buenos Aires: Manantial, 2008.

LEMKE, Thomas. New Materialisms: Foucault and the 'Government of Things'. Theory, Culture \& Society, vol. 32, n 4, p. 3-25, 1 jul. 2015.

LEVINAS, Emmanuel. Totalidad e infinito: ensayo sobre la exterioridad. Salamanca: Ediciones Sígueme, 2002.

LUPTON, Deborah. Self-tracking modes: Reflexive self-monitoring and data practices. SSRN Eletronic Journal, 2014. http://dx.doi.org/10.2139/ssrn.2483549

LUPTON, Deborah. The quantified self: a sociology of self-tracking. Cambridge: Polity, 2016a.

LUPTON, Deborah. Digital companion species and eating data: Implications for theorising digital data-human assemblages. Big Data \& Society, vol. 3, $\mathrm{n}^{\mathrm{o}} 1$, $\mathrm{p}$. $205395171561994,2016 b$.

LUPTON, Deborah. Data thing-power: how do personal digital data come to matter? SSRN Eletronic Journal, 2017. http://dx.doi.org/10.2139/ssrn.2998571

MOL, Annemarie. The body multiple: ontology in medical practice. Durham: Duke University Press, 2007.

NAFUS, Dawn.; SHERMAN, Jamie. Big Data, Big Questions| This One Does Not Go Up To 11: The Quantified Self Movement as an Alternative Big Data Practice. International Journal of Communication, vol. 8, $\mathrm{n}^{\circ}$ 0, p. 11, 2014. 
PFEIFER, Geoff. The new materialism: Althusser, Badiou, Žižek. New York: Routledge, 2015.

RABINOW, Paul. Essays on the anthropology of reason. Princeton: Princeton Univ. Press, 1996.

REIGELUTH, Tyler Butler. Why data is not enough: Digital traces as control of self and self-control. Surveillance \& Society, vol. 12, n 2, p. 243-254, 2014.

ROSE, Nikolas. Governing the soul: the shaping of the private self. London; New York: Routledge, 1990.

ROSE, Nikolas. Inventing our selves: psychology, power, and personhood. Cambridge, England; New York: Cambridge University Press, 1996.

ROSE, Nikolas. Políticas de la vida: biomedicina, poder y subjetividad en el siglo XXI. La Plata, Buenos Aires: UNIPE: Editorial Universitaria, 2012.

ROUVROY, Antoinette; BERNS, Thomas. Governamentalidade algorítmica e perspectivas de emancipação: o díspar como condição de individuação pela relação? Tradução de P. H. Andrade. Revista Eco Pós, vol. 18, no 2, p. 36-56, 2015.

RUCKENSTEIN, Minna.; PANTZAR, Mika. Beyond the Quantified Self: Thematic exploration of a dataistic paradigm. New Media \& Society, vol. 19, n 3, p. 401-418, 2017.

SANDERS, Rachel. Self-tracking in the digital era: biopower, patriarchy, and the new biometric body projects. Body \& Society, vol. 23, no 1, p. 36-63, 2017.

SWAN, Melanie. Health 2050: The Realization of Personalized Medicine through Crowdsourcing, the Quantified Self, and the Participatory Biocitizen. Journal of Personalized Medicine, vol. 2, nº 3, p. 93-118, 2012.

VAN DEN EEDE, Yoni. Tracing the tracker: a postphenomenological inquiry into selftracking technologies. Em: ROSENBERGER, Robert.; VERBEEK, Peter (Eds.). Postphenomenological Investigations: Essays on Human-Technology Relations. Lanham: Lexington Books, 2015. p. 143-158.

VERBEEK, Peter. What things do: philosophical reflections on technology, agency, and design. University Park, Penn.: Pennsylvania State University Press, 2005.

VERBEEK, Peter. Toward a Theory of Technological Mediation: A Program for Postphenomenological Research. Em: Technoscience and Postphenomenology: The Manhattan Papers. London: Lexington Books, 2016. p. 189-204. 
WHITSON, Jennifer. Gaming the quantified self. Surveillance \& Society, vol. 11, $\mathrm{n}^{\mathrm{o}}$ 1/2, $\mathrm{p}$. 163, 2013.

WOLF, Gary. The quantified self. Cannes, 2010. Disponível em: <https://www.ted.com/talks/gary_wolf_the_quantified_self>. Acesso em: 30 mar. 2017

Diego González Garcia Docente del Instituto de Fundamentos y Métodos de la Psicología, Facultad de Psicología de la Universidad de la República, Uruguay (UdelaR). Candidato a Doctor en "Persona y Sociedad en el Mundo Contemporáneo" de la Universidad Autónoma de Barcelona, España (UAB). Master en Psicología Social por la UdelaR. Integrante de los Grupos de investigación "Gubernamentalidad, Ciencia, Tecnología y Subjetivación" UdelaR y de "Psicopolitics Studies" UAB. ORCID: https://orcid.org/0000-0002-6648-8673 E-mail: $\underline{\text { dgonzalez@psico.edu.uy }}$

Marco Maureira Velasquez Doctor en Filosofía Contemporánea y Estudios Clásicos por la Universidad de Barcelona (UB) y Doctor en Psicología Social por la Universidad Autónoma de Barcelona (UAB). Ha desempeñado funciones como consultor en la División de Desarrollo Social de la Comisión Económica para América Latina y el Caribe (CEPALNaciones Unidas) y como colaborador en el Barcelona Science and Technology Studies Group (STS-b) y en el Grupo de Investigación en Filosofía Contemporánea, Ética y Política (APORÍA). ORCID: https://orcid.org/0000-0002-0238-6774 E-mail: maureira.marco@gmail.com

Francisco Tirado Serrano Profesor Titular del Departamento de Psicología Social de la Universidad Autónoma de Barcelona (UAB) Doctor en Psicología. Director del Doctorado "Persona y sociedad en el Mundo Contemporáneo" de la UAB. Coordinador de los grupos de Investigación Psicololitics Studies y del Barcelona Science and Technology Studies Group (STS-b). ORCID: https://orcid.org/0000-0001-7093-056X E-mail: FranciscoJavier.Tirado@uab.cat

1 Meetup es una aplicación del formato de red social cuyo eslogan es "somos lo que hacemos". Funciona como una plataforma para contactar a personas con los mismos intereses, crear eventos y grupos con actividades regulares donde los usuarios pueden participar y reunirse. 
2 Para más información, dirigirse a: https://medium.com/quantified-self-public-health

3 Esto no significa, en modo alguno, que los objetos técnicos sean completamente independientes del quehacer humano. Antes bien, el ser humano juega un rol de organización y coordinación en la dinámica técnica; a saber, "el hombre interviene como ser que regula su margen de indeterminación” (2007, p. 34), pero un margen -urge repetirlo-que es interior e inmanente al objeto mismo.

$4 \quad$ No olvidemos que, para Simondon, "la mayor causa de alienación en el mundo contemporáneo reside en este desconocimiento de la máquina" (2007, p. 31). 